[Chem. Pharm. Bull.

35( 5$) 1691-1701(1987)$

\title{
Crystal Structure of (Uracil-1-ylethyl)(adenin-9-ylethyl)tryptophan Dipeptide: An Interaction Model between Nucleic Acid Base and Aromatic Amino Acid ${ }^{11}$
}

\author{
Toshimasa Ishida, ${ }^{*}$ Yoshiko Tokura, Mari Shimamoto, \\ Mitsunobu Doi, and Masatoshi Inoue
}

Osaka University of Pharmaceutical Sciences, 2-10-65 Kawai, Matsubara, Osaka 580, Japan

(Received October 15, 1986)

\begin{abstract}
As a model compound for the interaction of tryptophan with nucleic acid bases, uracil and adenine, (uracil-1-ylethyl)(adenin-9-ylethyl)tryptophan dipeptide has been synthesized, and its molecular and crystal structure has been analyzed by the X-ray diffraction method. The crystal data are $a=4.989(2), b=30.056(5), c=9.939(1) \AA, \beta=94.61(3)^{\circ}, Z=2, D_{\mathrm{m}}=1.345(1) \mathrm{g} \cdot \mathrm{cm}^{-3}$, space group $P 2_{1}$. The molecule takes a $\mathrm{W}$-shaped extended conformation, and is linked with the neighboring molecule in a head-to-tail fashion through a reverse Watson-Crick base pairing. Consequently the molecules form an infinite chain along the $b$-axis. A weak stacking interaction is observed between the indole ring and the uracil base: the dihedral angle between them is $28.6(3)$, and the average interplanar spacing is $3.95 \AA$. This stacking mode is formed by the dipole-dipole coupling between the respective aromatic rings. The result may indicate a possible access mode of a tryptophan residue to the uracil base of a single-stranded nucleic acid.
\end{abstract}

Keywords - (uracil-1-ylethyl)(adenin-9-ylethyl)tryptophan dipeptide; X-ray analysis; indole-uracil stacking interaction; reverse Watson-Crick base pairing

Specific interaction between the chemical groups of amino acid and nucleic acid bases is important for the selective recognition of nucleic acid base sequences by proteins. Several types of specific interactions are possible, and tryptophan, which has the best $\pi$-electron donating character among aromatic amino acids, ${ }^{2)}$ has been shown to associate with nucleic acid bases by $\pi-\pi$ stacking interaction. ${ }^{3)}$ Since the stacking, in general, arises from the interaction between the $\pi$-orbitals of two aromatic rings, particularly from the coupling between the highest occupied molecular orbital (HOMO) of the indole ring (as a donor) and the lowest unoccupied one (LUMO) of the nucleic acid base (as an acceptor), ${ }^{4-6)}$ the interaction could be an important mode of selective recognition of nucleic acid bases by aromatic amino acids. At present, structural knowledge on the tryptophan-nucleic acid base stacking interaction has been mainly derived from solution studies using various spectroscopic methods, and few crystallographic studies have been done because of the difficulty of

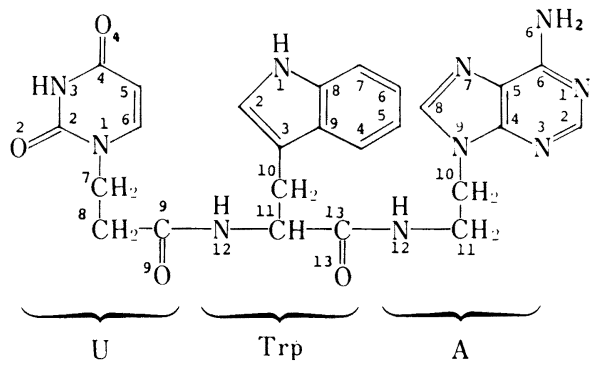

Fig. 1. Chemical Structure of U-Trp-A, and Atomic Numbering 
preparing suitable model crystals. However, information at the atomic level is absolutely necessary to establish the details of the interaction.

As a part of our structural studies on the interaction mode between indole ring and nucleic acid base, we have synthesized (uracil-1-ylethyl)(adenin-9-ylethyl)tryptophan dipeptide (U-Trp-A) (see Fig. 1 for its chemical structure), and analyzed the crystal structure by the X-ray diffraction method, in the expectation that this compound would provide information as to the binding geometry of tryptophan residue with adenine(A), uracil(U), or A $\cdots U$ base pair.

\section{Experimental}

Synthesis of U-Trp-A_- The synthetic scheme is presented in Fig. 2. Uracil-1-ylpropionic acid (1) and adenin9-ylethylamine (2) were synthesized from uracil and adenine, respectively, by methods similar to those described by Ueda and Fox, ${ }^{7)}$ and Leonard and Lambert. ${ }^{8)}$ U-Trp-A was then synthesized by the normal procedure of peptide synthesis. The crude products were purified by gel filtration on a Sephadex LH-20 column with methanol-water ( $7: 3$, $\mathrm{v} / \mathrm{v}$ ): Anal. Calcd for $\mathrm{C}_{25} \mathrm{H}_{26} \mathrm{~N}_{10} \mathrm{O}_{4} \cdot \mathrm{H}_{2} \mathrm{O}: \mathrm{C}, 54.74 ; \mathrm{H}, 5.14 ; \mathrm{N}, 25.53$. Found: C, 54.98; H, 5.64; N, 25.90.

X-Ray Data - Single crystals suitable for X-ray study were obtained from $50 \%$ aqueous methanol by the vapor diffusion technique. A single crystal, with dimensions of approx. $0.1 \times 0.3 \times 0.5 \mathrm{~mm}^{3}$, was used for the X-ray diffraction data collection. The crystallographic data are listed in Table $\mathrm{I}$. The intensity data were collected with a Rigaku AFC-5 diffractometer using graphite-monochromated $\mathrm{Cu} K_{\alpha}$ radiation at room temperature. A total of 2897 independent reflections within $\sin \theta / \hat{\lambda}=0.591 \AA^{-1}$ was collected in the $\omega-2 \theta$ scan mode and corrected for Lorentz and polarization factors, but not for absorption effects. The scan speed was $6 \%$ min and the background was taken for $3 \mathrm{~s}$ at both sides of every reflection.

Structure Determination and Refinement-The structure was solved by the direct method using the MULTAN78 program. ${ }^{9)}$ An E-map computed with the phase set of the highest figure of merit revealed the positions

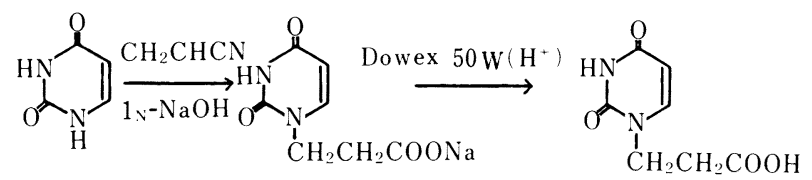

1

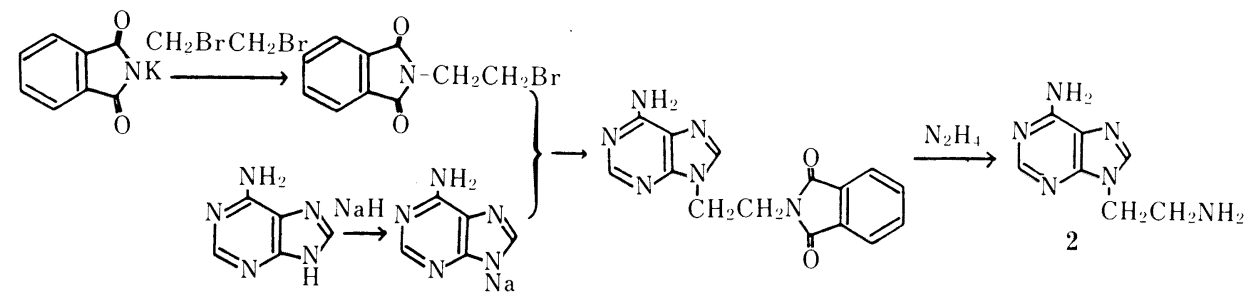

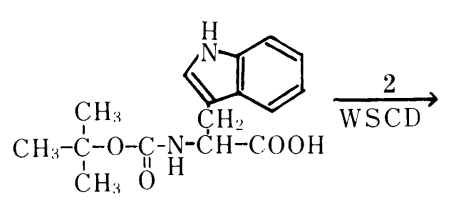<smiles>CC(C)(C)OC(=O)N[C@H](C(=O)O)c1c[nH]c2ccccc12</smiles><smiles></smiles>

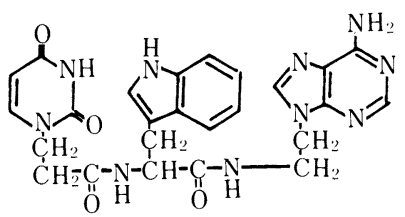

Fig. 2. Synthetic Scheme for U-Trp-A 
TABLE I. Crystal Data for U-Trp-A

\begin{tabular}{ll}
\hline \hline Molecular formula & $\mathrm{C}_{25} \mathrm{H}_{26} \mathrm{~N}_{10} \mathrm{O}_{4} \cdot 4 \mathrm{H}_{2} \mathrm{O}$ \\
Molecular weight & 602.61 \\
Crystal system & Monoclinic \\
Space group & $P 2_{1}$ \\
Cell constants & \\
$\quad a(\AA)$ & $4.989(2)$ \\
$\quad b(\AA)$ & $30.056(5)$ \\
$\quad c(\AA)$ & $9.939(1)$ \\
$\beta\left({ }^{\circ}\right)$ & $94.61(3)$ \\
Volume $\left(\AA^{3}\right)$ & $1485.6(6)$ \\
$Z$ & 2 \\
$\rho\left(\right.$ obsd.) $\left(\mathrm{g} \cdot \mathrm{cm}^{-3}\right)$ & $1.345(1)$ \\
$\rho$ (calcd.) & 1.347 \\
$F(000)$ & 636 \\
$\mu\left(\right.$ Cu $\left.K_{\alpha}\right)\left(\mathrm{cm}^{-1}\right)$ & 8.25 \\
\hline
\end{tabular}

of the $\mathrm{A}$ and $\mathrm{U}$ bases. The remaining atoms were positioned by successive Fourier syntheses. The structure was refined by the full-matrix least-squares method wih isotropic thermal parameters and then by the block-diagonal least-squares method with anisotropic ones. All the hydrogen atoms, except those of some molecules of water of crystallization, were revealed on a difference Fourier mpa (peak height: $0.3-0.5 e \AA^{-3}$ ). The final least-squares refinement including the hydrogen atoms with isotropic thermal parameters was computed to minimize $\sum w\left(\left|F_{\mathrm{o}}\right|-\left|F_{\mathrm{c}}\right|\right)^{2}$ with the following weighting scheme: $w=1.0 /\left[\sigma\left(F_{\mathrm{o}}\right)^{2}-0.02314\left|F_{\mathrm{o}}\right|+0.00622\left|F_{\mathrm{o}}\right|^{2}\right]$, where $\sigma\left(F_{\mathrm{o}}\right)$ is the standard deviation based on counting statistics. During the last cycle of refinement, none of the positional parameters shifted by more than one fourth of the estimated standard deviation. The final $R$ and $R w$ values using 2265 reflections having $F \geqq 3 \sigma\left(F_{\mathrm{o}}\right)$ are 0.07 and 0.09 , respectively. The residual fluctuations in the final difference Fourier map were within the range of $-0.3-0.3 e \AA^{-3}$. Final atomic coordinates of nonhydrogen atoms are given in Table II. ${ }^{10)}$

The structural refinements and other numerical calculations were carried out using the UNICS program ${ }^{11)}$ and the published scattering factors. ${ }^{12)}$ All calculations were carried out on an ACOS 900 computer of the Computer Center of Osaka University.

\section{Results and Discussion}

\section{Molecular Dimension and Conformation}

The bond lengths and angles for nonhydrogen atoms are given in Table III; the average bond distances involving hydrogen atoms are $\mathrm{C}-\mathrm{H}=1.08$ and $\mathrm{N}-\mathrm{H}=0.87 \AA$ with estimated standard deviations of $0.09 \AA$. A stereoscopic view of the U-Trp-A molecule is shown in Fig. 3 , and the conformational torsion angles are listed in Table IV.

Average bond lengths and angles for neutral $\mathrm{A}$ and $\mathrm{U}$ bases have been reported by Voet and Rich, ${ }^{13)}$ and are in good agreement with those of U-Trp-A within the standard errors. The molecular dimensions of the indole ring and the peptide moieties are also normal as judged from those of related indole ${ }^{14)}$ and peptide compounds. ${ }^{15)}$ The planarities of $\mathrm{U}$, indole, $\mathrm{A}$, and two peptides were examined in terms of the equations of least-squares planes. The results are summarized in Table $\mathrm{V}$. The rings of $\mathrm{U}$, indole and $\mathrm{A}$ are almost planar: maximum deviations are $-0.005 \AA$ for the $C(2)$ atom of $U,-0.035 \AA$ for the $C(5)$ atom of indole and $0.026 \AA$ for the $\mathrm{N}(7)$ atom of $\mathrm{A}$, and the atoms directly attached to the respective rings lay approximately on the planes. The two peptide linkages also form planes with trans conformations. The conformation of $\mathrm{U}-\operatorname{Trp}-\mathrm{A}$, as a whole, is depictable as a W-sharped, extended form. It is characteristic that the side rings of $\mathrm{U}$, indole and $\mathrm{A}$ all protrude to the same side of the peptide chain. In order to avoid close atomic contacts, $\mathrm{C}(8) \mathrm{U}$ and two $\mathrm{H}(7) \mathrm{U}$ atoms are in a staggered orientation with respect to the $\mathrm{C}(2) \mathrm{U}$ and $\mathrm{C}(6) \mathrm{U}$ atoms of the uracil base, and consequently the torsion angle $\chi_{2}$ is close to $90^{\circ}$. Similar conformations are also 
TABLE II. Final Atomic Coordinates of U-Trp-A

\begin{tabular}{|c|c|c|c|c|}
\hline Atom & $x$ & $y$ & $z$ & $B_{\mathrm{eq}}{ }^{a)}$ \\
\hline $\mathrm{N}(1) \mathrm{A}^{b)}$ & $0.527 \quad(1)$ & $0.2934(2)$ & 0.8768 & $3.7(2)$ \\
\hline$C(2) A$ & 0.539 & $0.3040(2)$ & $0.7473(6)$ & $3.6(2)$ \\
\hline $\mathrm{N}(3) \mathrm{A}$ & $0.7037(9)$ & $0.3325(2)$ & 0.6949 & $3.3(2)$ \\
\hline $\mathrm{C}(4) \mathrm{A}$ & $0.877 \quad(1)$ & $0.3513(2)$ & $0.7904(5)$ & $2.9(2)$ \\
\hline $\mathrm{C}(5) \mathrm{A}$ & $0.881 \quad$ (1) & $0.3437(2)$ & $0.9276(6)$ & $3.3(2)$ \\
\hline$C(6) A$ & $0.699 \quad(1)$ & $0.3126(2)$ & $0.9712(6)$ & $3.2(2)$ \\
\hline $\mathrm{N}(6) \mathrm{A}$ & 0.69 .2 & $0.2999(2)$ & $1.1012(5)$ & $4.6(3)$ \\
\hline$N(7) A$ & 1.081 & $0.3693(2)$ & $0.9952(5)$ & $3.8(2)$ \\
\hline $\mathrm{C}(8) \mathrm{A}$ & 1.192 & $0.3901(2)$ & $0.8984(6)$ & $3.7(2)$ \\
\hline $\mathrm{N}(9) \mathrm{A}$ & $1.0763(9)$ & $0.3800(2)$ & $0.7716(5)$ & $3.1(2)$ \\
\hline $\mathrm{C}(10) \mathrm{A}$ & $1.148 \quad(1)$ & $0.3998(2)$ & $0.6450(6)$ & $3.4(2)$ \\
\hline $\mathrm{C}(11) \mathrm{A}$ & $0.980 \quad(1)$ & $0.4412(2)$ & $0.6070(6)$ & $3.4(2)$ \\
\hline $\mathrm{N}(12) \mathrm{A}$ & $1.0118(9)$ & $0.4744(2)$ & $0.7146(5)$ & $3.5(2)$ \\
\hline $\mathrm{N}(1) \mathrm{T}$ & $0.893 \quad$ (2) & $0.5931(3)$ & $1.3014(7)$ & $6.4(3)$ \\
\hline $\mathrm{C}(2) \mathrm{T}$ & $0.742 \quad(2)$ & 0.5771 & $1.1868(7)$ & $5.5(4)$ \\
\hline$C(3) \mathrm{T}$ & $0.845 \quad(1)$ & 0.5384 & $1.1493(7)$ & $4.3(3)$ \\
\hline $\mathrm{C}(4) \mathrm{T}$ & $1.240 \quad(1)$ & $0.4912(3)$ & $1.2633(7)$ & $5.6(4)$ \\
\hline $\mathrm{C}(5) \mathrm{T}$ & $1.430 \quad(2)$ & $0.4901(4)$ & 1.3707 (9) & $7.3(5)$ \\
\hline $\mathrm{C}(6) \mathrm{T}$ & $1.452 \quad(2)$ & $0.5264(4)$ & 1.460 & $8.0(5)$ \\
\hline $\mathrm{C}(7) \mathrm{T}$ & $1.289 \quad(2)$ & 0.5619 & $1.4496(8)$ & $7.2(5)$ \\
\hline $\mathrm{C}(8) \mathrm{T}$ & $1.087 \quad(2)$ & 0.5632 & $1.3385(7)$ & $5.4(4)$ \\
\hline $\mathrm{C}(9) \mathrm{T}$ & $1.067 \quad(1)$ & 0.5278 & $1.2466(7)$ & $4.5(3)$ \\
\hline $\mathrm{C}(10) \mathrm{T}$ & $0.745 \quad(1)$ & $0.5095(2)$ & $1.0338(7)$ & $4.2(3)$ \\
\hline $\mathrm{C}(11) \mathrm{T}$ & $0.895 \quad$ (1) & $0.5177(2)$ & $0.9061(6)$ & $3.1(2)$ \\
\hline $\mathrm{N}(12) \mathrm{T}$ & $0.8298(8)$ & $0.5613(2)$ & $0.8487(5)$ & $3.1(2)$ \\
\hline $\mathrm{C}(13) \mathrm{T}$ & $0.823 \quad(1)$ & $0.4832(2)$ & $0.7967(6)$ & $3.1(2)$ \\
\hline $\mathrm{O}(13) \mathrm{T}$ & $0.5979(8)$ & $0.4664(2)$ & $0.7871(5)$ & $4.4(2)$ \\
\hline $\mathrm{N}(1) \mathrm{U}$ & $1.067 \quad(1)$ & $0.6862(2)$ & $0.9081(5)$ & $3.7(2)$ \\
\hline $\mathrm{C}(2) \mathrm{U}$ & $0.868 \quad(1)$ & $0.7155(2)$ & $0.9351(6)$ & $3.6(3)$ \\
\hline $\mathrm{O}(2) \mathrm{U}$ & $0.700 \quad(1)$ & 0.7292 & $0.8495(5)$ & $5.2(2)$ \\
\hline $\mathrm{N}(3) \mathrm{U}$ & $0.869 \quad(1)$ & $0.7287(2)$ & $1.0670(6)$ & $4.5(3)$ \\
\hline $\mathrm{C}(4) \mathrm{U}$ & $1.043 \quad(1)$ & $0.7152(3)$ & $1.1707(7)$ & $4.7(3)$ \\
\hline $\mathrm{O}(4) \mathrm{U}$ & $1.016 \quad(1)$ & $0.7301(2)$ & $1.2861(6)$ & $6.4(3)$ \\
\hline $\mathrm{C}(5) \mathrm{U}$ & $1.247 \quad(2)$ & 0.6841 & $1.1363(8)$ & $5.3(3)$ \\
\hline $\mathrm{C}(6) \mathrm{U}$ & 1.249 & $0.6713(2)$ & $1.0094(7)$ & $4.4(3)$ \\
\hline $\mathrm{C}(7) \mathrm{U}$ & $1.076 \quad(1)$ & $0.6718(2)$ & $0.7665(6)$ & $3.8(3)$ \\
\hline $\mathrm{C}(8) \mathrm{U}$ & $0.908 \quad$ (1) & $0.6306(2)$ & $0.7356(6)$ & $3.6(2)$ \\
\hline $\mathrm{C}(9) \mathrm{U}$ & 1.014 & $0.5890(2)$ & $0.8040(5)$ & $2.8(2)$ \\
\hline $\mathrm{O}(9) \mathrm{U}$ & $1.2557(8)$ & $0.5816(1)$ & $0.8204(5)$ & $4.1(2)$ \\
\hline $\mathrm{O}(1) \mathrm{W}$ & $0.104 \quad$ (1) & $0.3577(3)$ & $0.2837(5)$ & $7.7(3)$ \\
\hline $\mathrm{O}(2) \mathrm{W}$ & $0.377 \quad(1)$ & $0.8428(5)$ & $0.5896(6)$ & $12.2(6)$ \\
\hline $\mathrm{O}(3) \mathrm{W}$ & 0.299 & $0.1736(3)$ & $0.5647(8)$ & $11.0(5)$ \\
\hline $\mathrm{O}(4) \mathrm{W}$ & $1.365 \quad$ (3) & $0.7309(6)$ & 0.532 (1) & $18.0(1)$ \\
\hline
\end{tabular}

The estimated standard deviations are shown in parentheses.

a) $B_{\mathrm{eq}}=4 / 3\left(B_{11} a^{2}+B_{22} b^{2}+B_{33} c^{2}+a c B_{13} \cos \beta\right)$. b) The suffixes $\mathrm{A}, \mathrm{T}, \mathrm{U}$ and $\mathrm{W}$ represent the adenine, tryptophan and uracil moieties of U-Trp-A, and water of crystallization, respectively.

seen in the indole and adenine moieties. The tryptophan moiety takes a conformation that is commonly observed in related compounds ${ }^{16)}$ : the carbonyl and amino groups are in the trans and gauche ${ }^{-}$positions with respect to the indole ring $(\mathrm{C}(13) \mathrm{T}-\mathrm{C}(11) \mathrm{T}-\mathrm{C}(10)-\mathrm{C}(3) \mathrm{T}=$ $170.9(5)^{\circ}$ and $\left.\chi_{1}=-69.1(7)^{\circ}\right)$.

\section{Crystal Packing and Hydrogen Bonding Mode}

Figure 4 shows the crystal packings viewed along the $a$ and $c$ axes; molecules of water of 
TABLE III. Bond Lengths and Angles of U-Trp-A

1) Bond lengths $(\AA)$

\begin{tabular}{llll}
\hline \multicolumn{1}{c}{ Bond } & Distance & \multicolumn{1}{c}{ Bond } & Distance \\
\hline $\mathrm{N}(1) \mathrm{A}-\mathrm{C}(2) \mathrm{A}$ & $1.332(8)$ & $\mathrm{C}(5) \mathrm{T}-\mathrm{C}(6) \mathrm{T}$ & $1.41(2)$ \\
$\mathrm{N}(1) \mathrm{A}-\mathrm{C}(6) \mathrm{A}$ & $1.348(8)$ & $\mathrm{C}(6) \mathrm{T}-\mathrm{C}(7) \mathrm{T}$ & $1.34(2)$ \\
$\mathrm{C}(2) \mathrm{A}-\mathrm{N}(3) \mathrm{A}$ & $1.323(8)$ & $\mathrm{C}(7) \mathrm{T}-\mathrm{C}(8) \mathrm{T}$ & $1.43(2)$ \\
$\mathrm{N}(3) \mathrm{A}-\mathrm{C}(4) \mathrm{A}$ & $1.355(8)$ & $\mathrm{C}(8) \mathrm{T}-\mathrm{C}(9) \mathrm{T}$ & $1.40(1)$ \\
$\mathrm{C}(4) \mathrm{A}-\mathrm{C}(5) \mathrm{A}$ & $1.381(8)$ & $\mathrm{C}(10) \mathrm{T}-\mathrm{C}(11) \mathrm{T}$ & $1.547(9)$ \\
$\mathrm{C}(4) \mathrm{A}-\mathrm{N}(9) \mathrm{A}$ & $1.342(7)$ & $\mathrm{C}(11) \mathrm{T}-\mathrm{N}(12) \mathrm{T}$ & $1.457(8)$ \\
$\mathrm{C}(5) \mathrm{A}-\mathrm{C}(6) \mathrm{A}$ & $1.399(8)$ & $\mathrm{C}(11) \mathrm{T}-\mathrm{C}(13) \mathrm{T}$ & $1.524(8)$ \\
$\mathrm{C}(5) \mathrm{A}-\mathrm{N}(7) \mathrm{A}$ & $1.390(8)$ & $\mathrm{N}(12) \mathrm{T}-\mathrm{C}(9) \mathrm{U}$ & $1.344(7)$ \\
$\mathrm{C}(6) \mathrm{A}-\mathrm{N}(6) \mathrm{A}$ & $1.349(8)$ & $\mathrm{C}(13) \mathrm{T}-\mathrm{O}(13) \mathrm{T}$ & $1.229(8)$ \\
$\mathrm{N}(7) \mathrm{A}-\mathrm{C}(8) \mathrm{A}$ & $1.308(8)$ & $\mathrm{N}(1) \mathrm{U}-\mathrm{C}(2) \mathrm{U}$ & $1.367(8)$ \\
$\mathrm{C}(8) \mathrm{A}-\mathrm{N}(9) \mathrm{A}$ & $1.379(8)$ & $\mathrm{N}(1) \mathrm{U}-\mathrm{C}(6) \mathrm{U}$ & $1.377(9)$ \\
$\mathrm{N}(9) \mathrm{A}-\mathrm{C}(10) \mathrm{A}$ & $1.462(8)$ & $\mathrm{N}(1) \mathrm{U}-\mathrm{C}(7) \mathrm{U}$ & $1.477(8)$ \\
$\mathrm{C}(10) \mathrm{A}-\mathrm{C}(11) \mathrm{A}$ & $1.534(9)$ & $\mathrm{C}(2) \mathrm{U}-\mathrm{O}(2) \mathrm{U}$ & $1.217(8)$ \\
$\mathrm{C}(11) \mathrm{A}-\mathrm{N}(12) \mathrm{A}$ & $1.462(8)$ & $\mathrm{C}(2) \mathrm{U}-\mathrm{N}(3) \mathrm{U}$ & $1.369(9)$ \\
$\mathrm{N}(12) \mathrm{A}-\mathrm{C}(13) \mathrm{T}$ & $1.320(8)$ & $\mathrm{N}(3) \mathrm{U}-\mathrm{C}(4) \mathrm{U}$ & $1.36(1)$ \\
$\mathrm{N}(1) \mathrm{T}-\mathrm{C}(2) \mathrm{T}$ & $1.40(1)$ & $\mathrm{C}(4) \mathrm{U}-\mathrm{O}(4) \mathrm{U}$ & $1.25(1)$ \\
$\mathrm{N}(1) \mathrm{T}-\mathrm{C}(8) \mathrm{T}$ & $1.35(1)$ & $\mathrm{C}(4) \mathrm{U}-\mathrm{C}(5) \mathrm{U}$ & $1.44(1)$ \\
$\mathrm{C}(2) \mathrm{T}-\mathrm{C}(3) \mathrm{T}$ & $1.34(1)$ & $\mathrm{C}(5) \mathrm{U}-\mathrm{C}(6) \mathrm{U}$ & $1.32(1)$ \\
$\mathrm{C}(3) \mathrm{T}-\mathrm{C}(9) \mathrm{T}$ & $1.45(1)$ & $\mathrm{C}(7) \mathrm{U}-\mathrm{C}(8) \mathrm{U}$ & $1.515(9)$ \\
$\mathrm{C}(3) \mathrm{T}-\mathrm{C}(10) \mathrm{T}$ & $1.50(1)$ & $\mathrm{C}(8) \mathrm{U}-\mathrm{C}(9) \mathrm{U}$ & $1.498(8)$ \\
$\mathrm{C}(4) \mathrm{T}-\mathrm{C}(5) \mathrm{T}$ & $1.37(2)$ & $\mathrm{C}(9) \mathrm{U}-\mathrm{O}(9) \mathrm{U}$ & $1.223(7)$ \\
$\mathrm{C}(4) \mathrm{T}-\mathrm{C}(9) \mathrm{T}$ & $1.40(1)$ & & \\
\hline
\end{tabular}

2) Bond angles ( $\left.{ }^{\circ}\right)$

\begin{tabular}{clll}
\hline \hline Bond & Angle & \multicolumn{1}{c}{ Bond } & Angle \\
\hline C(2)A-N(1)A-C(6)A & $119.7(5)$ & $\mathrm{N}(1) \mathrm{T}-\mathrm{C}(8) \mathrm{T}-\mathrm{C}(9) \mathrm{T}$ & $108.4(7)$ \\
N(1)A-C(2)A-N(3)A & $127.8(6)$ & $\mathrm{C}(7) \mathrm{T}-\mathrm{C}(8) \mathrm{T}-\mathrm{C}(9) \mathrm{T}$ & $119.5(8)$ \\
$\mathrm{C}(2) \mathrm{A}-\mathrm{N}(3) \mathrm{A}-\mathrm{C}(4) \mathrm{A}$ & $112.1(5)$ & $\mathrm{C}(3) \mathrm{T}-\mathrm{C}(9) \mathrm{T}-\mathrm{C}(4) \mathrm{T}$ & $133.3(8)$ \\
N(3)A-C(4)A-C(5)A & $125.6(5)$ & $\mathrm{C}(3) \mathrm{T}-\mathrm{C}(9) \mathrm{T}-\mathrm{C}(8) \mathrm{T}$ & $106.2(7)$ \\
N(3)A-C(4)A-N(9)A & $127.5(5)$ & $\mathrm{C}(4) \mathrm{T}-\mathrm{C}(9) \mathrm{T}-\mathrm{C}(8) \mathrm{T}$ & $120.5(8)$ \\
$\mathrm{C}(5) \mathrm{A}-\mathrm{C}(4) \mathrm{A}-\mathrm{N}(9) \mathrm{A}$ & $106.9(5)$ & $\mathrm{C}(3) \mathrm{T}-\mathrm{C}(10) \mathrm{T}-\mathrm{C}(11) \mathrm{T}$ & $112.5(6)$ \\
$\mathrm{C}(4) \mathrm{A}-\mathrm{C}(5) \mathrm{A}-\mathrm{C}(6) \mathrm{A}$ & $117.2(5)$ & $\mathrm{C}(10) \mathrm{T}-\mathrm{C}(11) \mathrm{T}-\mathrm{N}(12) \mathrm{T}$ & $110.8(5)$ \\
$\mathrm{C}(4) \mathrm{A}-\mathrm{C}(5) \mathrm{A}-\mathrm{N}(7) \mathrm{A}$ & $109.9(5)$ & $\mathrm{C}(10) \mathrm{T}-\mathrm{C}(11) \mathrm{T}-\mathrm{C}(13) \mathrm{T}$ & $112.0(5)$ \\
$\mathrm{C}(6) \mathrm{A}-\mathrm{C}(5) \mathrm{A}-\mathrm{N}(7) \mathrm{A}$ & $132.9(5)$ & $\mathrm{N}(12) \mathrm{T}-\mathrm{C}(11) \mathrm{T}-\mathrm{C}(13) \mathrm{T}$ & $107.5(5)$ \\
N(1)A-C(6)A-C(5)A & $117.6(5)$ & $\mathrm{C}(11) \mathrm{T}-\mathrm{N}(12) \mathrm{T}-\mathrm{C}(9) \mathrm{U}$ & $123.3(5)$ \\
N(1)A-C(6)A-N(6)A & $118.8(5)$ & $\mathrm{N}(12) \mathrm{A}-\mathrm{C}(13) \mathrm{T}-\mathrm{C}(11) \mathrm{T}$ & $115.9(5)$ \\
$\mathrm{C}(5) \mathrm{A}-\mathrm{C}(6) \mathrm{A}-\mathrm{N}(6) \mathrm{A}$ & $123.6(5)$ & $\mathrm{N}(12) \mathrm{A}-\mathrm{C}(13) \mathrm{T}-\mathrm{O}(13) \mathrm{T}$ & $124.3(6)$ \\
$\mathrm{C}(5) \mathrm{A}-\mathrm{N}(7) \mathrm{A}-\mathrm{C}(8) \mathrm{A}$ & $103.9(5)$ & $\mathrm{C}(11) \mathrm{T}-\mathrm{C}(13) \mathrm{T}-\mathrm{O}(13) \mathrm{T}$ & $119.8(5)$ \\
N(7)A-C(8)A-N(9)A & $113.3(6)$ & $\mathrm{C}(2) \mathrm{U}-\mathrm{N}(1) \mathrm{U}-\mathrm{C}(6) \mathrm{U}$ & $120.9(5)$ \\
$\mathrm{C}(4) \mathrm{A}-\mathrm{N}(9) \mathrm{A}-\mathrm{C}(8) \mathrm{A}$ & $106.0(5)$ & $\mathrm{C}(2) \mathrm{U}-\mathrm{N}(1) \mathrm{U}-\mathrm{C}(7) \mathrm{U}$ & $117.0(5)$ \\
$\mathrm{C}(4) \mathrm{A}-\mathrm{N}(9) \mathrm{A}-\mathrm{C}(10) \mathrm{A}$ & $128.2(5)$ & $\mathrm{C}(6) \mathrm{U}-\mathrm{N}(1) \mathrm{U}-\mathrm{C}(7) \mathrm{U}$ & $122.1(5)$ \\
$\mathrm{C}(8) \mathrm{A}-\mathrm{N}(9) \mathrm{A}-\mathrm{C}(10) \mathrm{A}$ & $125.6(5)$ & $\mathrm{N}(1) \mathrm{U}-\mathrm{C}(2) \mathrm{U}-\mathrm{O}(2) \mathrm{U}$ & $123.4(6)$ \\
N(9)A-C(10)A-C(11)A & $112.0(5)$ & $\mathrm{N}(1) \mathrm{U}-\mathrm{C}(2) \mathrm{U}-\mathrm{N}(3) \mathrm{U}$ & $115.3(6)$ \\
$\mathrm{C}(10) \mathrm{A}-\mathrm{C}(11) \mathrm{A}-\mathrm{N}(12) \mathrm{A}$ & $110.4(5)$ & $\mathrm{O}(2) \mathrm{U}-\mathrm{C}(2) \mathrm{U}-\mathrm{N}(3) \mathrm{U}$ & $121.3(6)$ \\
$\mathrm{C}(11) \mathrm{A}-\mathrm{N}(12) \mathrm{A}-\mathrm{C}(13) \mathrm{T}$ & $123.1(5)$ & $\mathrm{C}(2) \mathrm{U}-\mathrm{N}(3) \mathrm{U}-\mathrm{C}(4) \mathrm{U}$ & $126.4(6)$ \\
$\mathrm{C}(2) \mathrm{T}-\mathrm{N}(1) \mathrm{T}-\mathrm{C}(8) \mathrm{T}$ & $108.7(7)$ & $\mathrm{N}(3) \mathrm{U}-\mathrm{C}(4) \mathrm{U}-\mathrm{O}(4) \mathrm{U}$ & $118.9(7)$ \\
N(1)T-C(2)T-C(3)T & $109.3(7)$ & $\mathrm{N}(3) \mathrm{U}-\mathrm{C}(4) \mathrm{U}-\mathrm{C}(5) \mathrm{U}$ & $115.8(7)$ \\
$\mathrm{C}(2) \mathrm{T}-\mathrm{C}(3) \mathrm{T}-\mathrm{C}(9) \mathrm{T}$ & $107.3(7)$ & $\mathrm{O}(4) \mathrm{U}-\mathrm{C}(4) \mathrm{U}-\mathrm{C}(5) \mathrm{U}$ & $125.4(7)$ \\
$\mathrm{C}(2) \mathrm{T}-\mathrm{C}(3) \mathrm{T}-\mathrm{C}(10) \mathrm{T}$ & $127.4(7)$ & $\mathrm{C}(4) \mathrm{U}-\mathrm{C}(5) \mathrm{U}-\mathrm{C}(6) \mathrm{U}$ & $118.3(7)$ \\
$\mathrm{C}(9) \mathrm{T}-\mathrm{C}(3) \mathrm{T}-\mathrm{C}(10) \mathrm{T}$ & $125.2(7)$ & $\mathrm{N}(1) \mathrm{U}-\mathrm{C}(6) \mathrm{U}-\mathrm{C}(5) \mathrm{U}$ & $123.2(7)$ \\
\hline
\end{tabular}


2) Bond angles $\left(^{\circ}\right)$

\begin{tabular}{clll}
\hline \hline Bond & Angle & \multicolumn{1}{c}{ Bond } & Angle \\
\hline $\mathrm{C}(5) \mathrm{T}-\mathrm{C}(4) \mathrm{T}-\mathrm{C}(9) \mathrm{T}$ & $119.4(9)$ & $\mathrm{N}(1) \mathrm{U}-\mathrm{C}(7) \mathrm{U}-\mathrm{C}(8) \mathrm{U}$ & $112.0(5)$ \\
$\mathrm{C}(4) \mathrm{T}-\mathrm{C}(5) \mathrm{T}-\mathrm{C}(6) \mathrm{T}$ & $119(1)$ & $\mathrm{C}(7) \mathrm{U}-\mathrm{C}(8) \mathrm{U}-\mathrm{C}(9) \mathrm{U}$ & $114.8(5)$ \\
$\mathrm{C}(5) \mathrm{T}-\mathrm{C}(6) \mathrm{T}-\mathrm{C}(7) \mathrm{T}$ & $124(1)$ & $\mathrm{N}(12) \mathrm{T}-\mathrm{C}(9) \mathrm{U}-\mathrm{C}(8) \mathrm{U}$ & $116.1(5)$ \\
$\mathrm{C}(6) \mathrm{T}-\mathrm{C}(7) \mathrm{T}-\mathrm{C}(8) \mathrm{T}$ & $118(1)$ & $\mathrm{N}(12) \mathrm{T}-\mathrm{C}(9) \mathrm{U}-\mathrm{O}(9) \mathrm{U}$ & $122.4(5)$ \\
$\mathrm{N}(1) \mathrm{T}-\mathrm{C}(8) \mathrm{T}-\mathrm{C}(7) \mathrm{T}$ & $132.1(9)$ & $\mathrm{C}(8) \mathrm{U}-\mathrm{C}(9) \mathrm{U}-\mathrm{O}(9) \mathrm{U}$ & $121.5(5)$ \\
\hline
\end{tabular}

The estimated standard deviations are shown in parentheses.
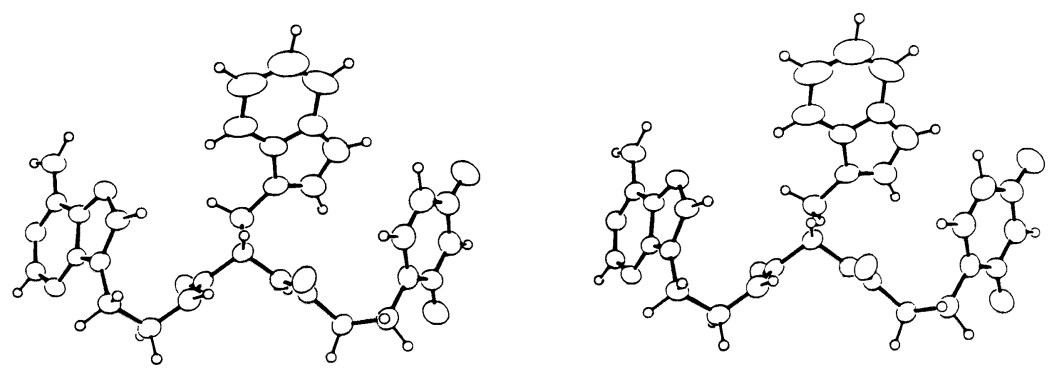

Fig. 3. A Stereoscopic View of U-Trp-A

TABLE IV. Selected Torsion Angles ( ${ }^{\cup}$ ) of U-Trp-A

\begin{tabular}{cclr}
\hline \hline $\mathrm{U}$ & $\psi$ & {$[\mathrm{C}(7) \mathrm{U}-\mathrm{C}(8) \mathrm{U}-\mathrm{C}(9) \mathrm{U}-\mathrm{N}(12) \mathrm{T}]$} & $141.7(5)$ \\
& $\omega$ & {$[\mathrm{C}(8) \mathrm{U}-\mathrm{C}(9) \mathrm{U}-\mathrm{N}(12) \mathrm{T}-\mathrm{C}(11) \mathrm{T}]$} & $174.1(5)$ \\
& $\chi_{1}$ & {$[\mathrm{C}(9) \mathrm{U}-\mathrm{C}(8) \mathrm{U}-\mathrm{C}(7) \mathrm{U}-\mathrm{N}(1) \mathrm{U}]$} & $-68.5(7)$ \\
& $\chi_{2}$ & {$[\mathrm{C}(8) \mathrm{U}-\mathrm{C}(7) \mathrm{U}-\mathrm{N}(1) \mathrm{U}-\mathrm{C}(2) \mathrm{U}]$} & $-89.8(6)$ \\
$\mathrm{T}$ Trp & $\phi$ & {$[\mathrm{C}(9) \mathrm{U}-\mathrm{N}(12) \mathrm{T}-\mathrm{C}(11) \mathrm{T}-\mathrm{C}(13) \mathrm{T}]$} & $-100.8(6)$ \\
& $\psi$ & {$[\mathrm{N}(12) \mathrm{T}-\mathrm{C}(11) \mathrm{T}-\mathrm{C}(13) \mathrm{T}-\mathrm{N}(12) \mathrm{A}]$} & $87.0(6)$ \\
& $\omega$ & {$[\mathrm{C}(11) \mathrm{T}-\mathrm{C}(13) \mathrm{T}-\mathrm{N}(12) \mathrm{A}-\mathrm{C}(11) \mathrm{A}]$} & $-69.1(7)$ \\
& $\chi_{1}$ & {$[\mathrm{~N}(12) \mathrm{T}-\mathrm{C}(11) \mathrm{T}-\mathrm{C}(10) \mathrm{T}-\mathrm{C}(3) \mathrm{T}]$} & $96.3(9)$ \\
& $\chi_{2}$ & {$[\mathrm{C}(11) \mathrm{T}-\mathrm{C}(10) \mathrm{T}-\mathrm{C}(3) \mathrm{T}-\mathrm{C}(2) \mathrm{T}]$} & $-106.5(6)$ \\
& $\phi$ & {$[\mathrm{C}(13) \mathrm{T}-\mathrm{N}(12) \mathrm{A}-\mathrm{C}(11) \mathrm{A}-\mathrm{C}(10) \mathrm{A}]$} & $57.9(6)$ \\
& $\chi_{1}$ & {$[\mathrm{~N}(12) \mathrm{A}-\mathrm{C}(11) \mathrm{A}-\mathrm{C}(10) \mathrm{A}-\mathrm{N}(9) \mathrm{A}]$} & $-90.7(7)$ \\
\hline
\end{tabular}

The estimated standard deviations are shown in parentheses.

crystallization are indicated by filled circles, and the dotted lines represent possible hydrogen bonds. The possible hydrogen bonds and short contacts observed in the crystal are summarized in Table VI. The polar atoms of the U-Trp-A molecule all participate in hydrogen bonds. The $\mathrm{N}(12) \mathrm{T}-\mathrm{O}(9) \mathrm{U}$ and $\mathrm{N}(12) \mathrm{A}-\mathrm{O}(13) \mathrm{T}$ atomic pairs of two peptide groups are hydrogen-bonded to those of the neighboring molecules related by $a$-axis translation, consequently forming an infinite layer along this axis (see Fig. 4(A)). On the other hand, U-Trp-A molecules related by a diad screw symmetry are linked in a head-to-tail fashion by two hydrogen bonds between the A and U bases (see Fig. 4(B)): two hydrogen bonds, $\mathrm{N}(1) \mathrm{A} \cdots \mathrm{N}(3) \mathrm{U}(=2.858(8) \AA)$ and $\mathrm{N}(6) \mathrm{A} \cdots \mathrm{O}(2) \mathrm{U}(=2.955(8) \AA)$ atomic pairs, form a reversed Watson-Crick base pairing. Similar base pairing has also been observed in the 8 bromo-9-ethyladenine: 1-methyl-5-bromouracil complex. ${ }^{17)}$

Four molecules of water of crystallization, which exist near the base pairing parts, 


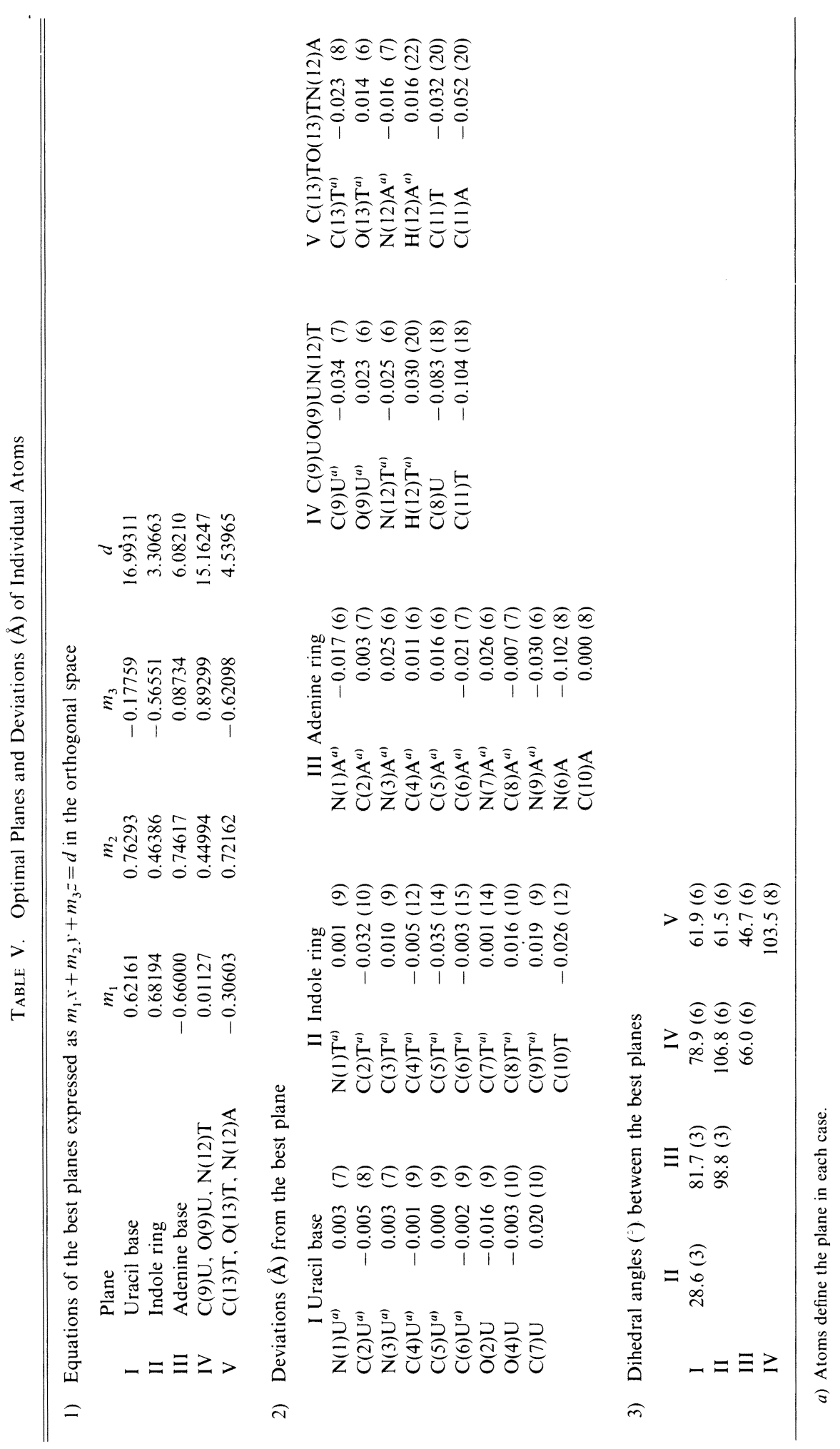


A

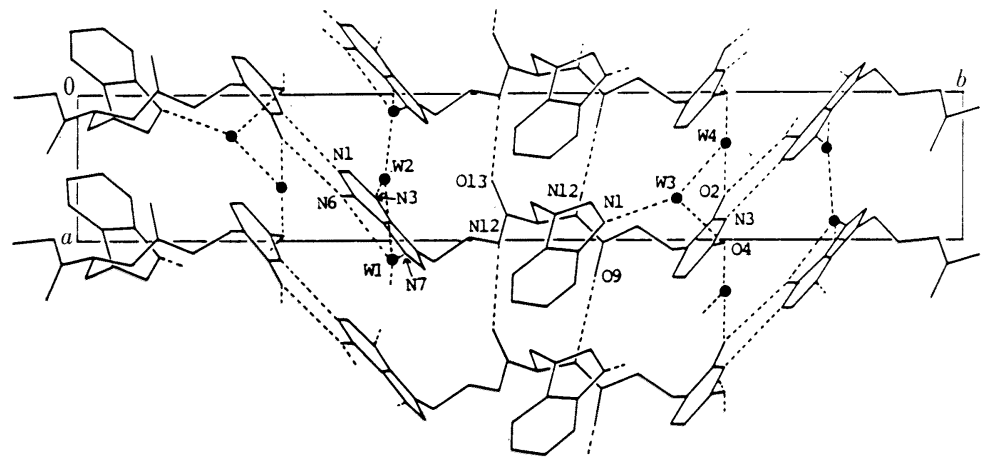

B

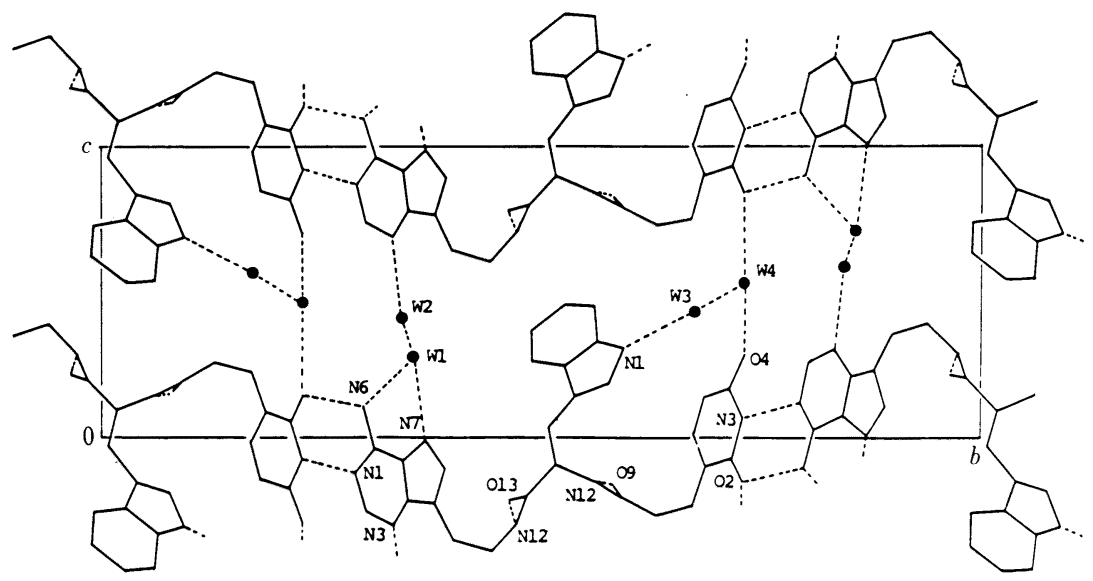

Fig. 4. Crystal Packing of U-Trp-A, Viewed along the $c$-Axis (A) and the $a$-Axis (B)

stabilize the molecular packing in the $c$ direction through hydrogen bonds with polar atoms of the aromatic rings.

\section{Stacking Interactions between the Aromatic Rings}

One of the structural characteristics observed in this crystal is the presence of four kinds of stacking interactions between the aromatic rings.

1) Stacking Interactions between Adenine Rings and between Indole Rings - - Stacking interactions between the adenine rings and between the indole rings are formed in the infinite layers of U-Trp-A arranged along the $a$-axis. The stacking modes are shown in Fig. 5, (A) and (B). The stacking rings are parallel to each other, and the average interplanar spacings are $3.25 \AA$ for the adenine rings and $3.43 \AA$ for the indole rings. These partial overlaps would be stabilized by electrostatic interactions in addition to the crystal packing forces. A comparison of the atomic charges (in atomic units), calculated by the $\mathrm{CNDO} / 2$ method, ${ }^{18)}$ indicates the electrostatic attraction between atomic pairs showing the short contacts (Table VI): $-0.283-$ 0.165 for $\mathrm{N}(1) \mathrm{A}-\mathrm{C}(8) \mathrm{A}, 0.214--0.122$ for $\mathrm{C}(2) \mathrm{A}-\mathrm{N}(9) \mathrm{A},-0.257-0.086$ for $\mathrm{N}(3) \mathrm{A}-$ $\mathrm{C}(10) \mathrm{A}, 0.024-0.158$ for $\mathrm{C}(6) \mathrm{T}-\mathrm{N}(1) \mathrm{T}$ and $0.005-0.035$ for $\mathrm{C}(5) \mathrm{T}-\mathrm{C}(3) \mathrm{T}$ atomic pairs.

2) Stacking Interaction between Adenine and Uracil Bases and between Uracil Base and Indole Ring Although we expected this compound to be an interaction model of 
TABLE VI. Hydrogen Bonds and Short Contacts

1) Hydrogen bonds

\begin{tabular}{|c|c|c|c|c|}
\hline Donor (D) at $x, y, z$ & Acceptor (A) & t symmetry operation & $\begin{array}{c}\text { Distance }(\AA) \\
\text { D } \cdots A\end{array}$ & $\begin{array}{l}\text { Angle }\left(^{\circ}\right) \\
\text { D-H } \cdots \text { A }\end{array}$ \\
\hline$N(3) U$ & $\mathrm{~N}(1) \mathrm{A}$ & $1-x, y+0.5,2-z$ & $2.858 \quad(8)$ & $175(4)$ \\
\hline $\mathrm{N}(1) \mathrm{T}$ & $\mathrm{O}(3) \mathrm{W}$ & $1-x, y+0.5,2-z$ & $2.957(13)$ & $165(6)$ \\
\hline $\mathrm{N}(12) \mathrm{T}$ & $\mathrm{O}(9) \mathrm{U}$ & $x-1, y, z$ & $2.919 \quad(7)$ & $172(5)$ \\
\hline $\mathrm{N}(6) \mathrm{A}$ & $\mathrm{O}(2) \mathrm{U}$ & $1-x, y-0.5,2-z$ & $2.955 \quad(8)$ & $172(4)$ \\
\hline$N(6) A$ & $\mathrm{O}(1) \mathrm{W}$ & $1+x, y, 1+z$ & $3.152(11)$ & $156(6)$ \\
\hline$N(12) A$ & $\mathrm{O}(13) \mathrm{T}$ & $1+x, y, z$ & $2.965 \quad(7)$ & $130(4)$ \\
\hline $\mathrm{O}(1) \mathrm{W}$ & $N(7) A$ & $x-1, y, z-1$ & $2.882(11)$ & $178(6)$ \\
\hline $\mathrm{O}(1) \mathrm{W}$ & $\mathrm{O}(2) \mathrm{W}$ & $1-x, y-0.5,1-z$ & $2.825(17)$ & - \\
\hline $\mathrm{O}(2) \mathrm{W}$ & $N(3) A$ & $1-x, y+0.5,1-z$ & $2.841(15)$ & $178(7)$ \\
\hline $\mathrm{O}(2) \mathrm{W}$ & $\mathrm{O}(1) \mathrm{W}$ & $-x, y+0.5,1-z$ & $2.831(17)$ & - \\
\hline $\mathrm{O}(3) \mathrm{W}$ & $\mathrm{O}(4) \mathrm{U}$ & $1-x, y-0.5,2-z$ & $2.813(13)$ & $177(6)$ \\
\hline $\mathrm{O}(3) \mathrm{W}$ & $\mathrm{O}(4) \mathrm{W}$ & $2-x, y-0.5,1-z$ & $2.639(21)$ & - \\
\hline $\mathrm{O}(4) \mathrm{W}$ & $\mathrm{O}(4) \mathrm{U}$ & $x, y, z-1$ & $2.881(19)$ & $180(7)$ \\
\hline $\mathrm{O}(4) \mathrm{W}$ & $\mathrm{O}(2) \mathrm{U}$ & $x+1, y, z$ & $3.454(19)$ & $178(7)$ \\
\hline
\end{tabular}

2) Short contacts (less than $3.5 \AA$ )

\begin{tabular}{llll|llll}
\hline \hline $\begin{array}{c}\text { Atom at } \\
x, y, z\end{array}$ & Atom at symmetry operation & $\begin{array}{c}\text { Distance } \\
(\AA)\end{array}$ & $\begin{array}{c}\text { Atom at } \\
x, y, z\end{array}$ & Atom at symmetry operation & $\begin{array}{c}\text { Distance } \\
(\AA)\end{array}$ \\
\hline $\mathrm{C}(8) \mathrm{U}$ & $\mathrm{O}(3) \mathrm{W}$ & $1-x, y+0.5,1-z$ & $3.339(12)$ & $\mathrm{N}(1) \mathrm{A}$ & $\mathrm{N}(7) \mathrm{A}$ & $x-1, y, z$ & $3.458(7)$ \\
$\mathrm{N}(1) \mathrm{A}$ & $\mathrm{C}(8) \mathrm{A}$ & $x-1, y, z$ & $3.369(8)$ & $\mathrm{C}(2) \mathrm{A}$ & $\mathrm{N}(9) \mathrm{A}$ & $x-1, y, z$ & $3.268(8)$ \\
$\mathrm{N}(3) \mathrm{A}$ & $\mathrm{C}(10) \mathrm{A}$ & $x-1, y, z$ & $3.434(8)$ & $\mathrm{C}(6) \mathrm{A}$ & $\mathrm{C}(8) \mathrm{A}$ & $x-1, y, z$ & $3.471(9)$ \\
$\mathrm{N}(1) \mathrm{T}$ & $\mathrm{C}(6) \mathrm{T}$ & $x-1, y, z$ & $3.451(15)$ & $\mathrm{C}(3) \mathrm{T}$ & $\mathrm{C}(5) \mathrm{T}$ & $x-1, y, z$ & $3.461(15)$ \\
$\mathrm{O}(13) \mathrm{T}$ & $\mathrm{C}(8) \mathrm{A}$ & $x-1, y, z$ & $3.307(8)$ & $\mathrm{O}(13) \mathrm{T}$ & $\mathrm{C}(10) \mathrm{A}$ & $x-1, y, z$ & $3.245(8)$ \\
$\mathrm{C}(2) \mathrm{U}$ & $\mathrm{C}(6) \mathrm{U}$ & $x-1, y, z$ & $3.492(10)$ & $\mathrm{O}(2) \mathrm{U}$ & $\mathrm{C}(6) \mathrm{U}$ & $x-1, y, z$ & $3.346(9)$ \\
$\mathrm{N}(3) \mathrm{U}$ & $\mathrm{C}(5) \mathrm{U}$ & $x-1, y, z$ & $3.495(10)$ & $\mathrm{O}(2) \mathrm{O}$ & $\mathrm{O}(4) \mathrm{W}$ & $x-1, y, z$ & $3.413(23)$ \\
$\mathrm{C}(4) \mathrm{U}$ & $\mathrm{O}(3) \mathrm{W}$ & $1-x, y+0.5,2-z$ & $3.478(13)$ & $\mathrm{N}(6) \mathrm{A}$ & $\mathrm{O}(2) \mathrm{W}$ & $1-x, y-0.5,2-z$ & $3.376(15)$ \\
$\mathrm{N}(1) \mathrm{A}$ & $\mathrm{C}(4) \mathrm{U}$ & $2-x, y-0.5,2-z$ & $3.244(9)$ & $\mathrm{N}(1) \mathrm{A}$ & $\mathrm{O}(4) \mathrm{U}$ & $2-x, y-0.5,2-z$ & $3.469(8)$ \\
$\mathrm{N}(1) \mathrm{A}$ & $\mathrm{C}(5) \mathrm{U}$ & $2-x, y-0.5,2-z$ & $3.477(10)$ & $\mathrm{C}(2) \mathrm{A}$ & $\mathrm{C}(4) \mathrm{U}$ & $2-x, y-0.5,2-z$ & $3.448(10)$ \\
$\mathrm{C}(2) \mathrm{A}$ & $\mathrm{O}(4) \mathrm{U}$ & $2-x, y-0.5,2-z$ & $3.179(9)$ & $\mathrm{N}(3) \mathrm{A}$ & $\mathrm{O}(4) \mathrm{U}$ & $2-x, y-0.5,2-z$ & $3.379(8)$ \\
$\mathrm{C}(6) \mathrm{A}$ & $\mathrm{N}(3) \mathrm{U}$ & $2-x, y-0.5,2-z$ & $3.360(8)$ & $\mathrm{N}(6) \mathrm{A}$ & $\mathrm{C}(2) \mathrm{U}$ & $2-x, y-0.5,2-z$ & 3.394 \\
\end{tabular}

tryptophan with the A-U Watson-Crick base pairing, the crystal structure showed an extended molecular packing different from that of a self-complementary dinucleotide such as $\mathrm{ApU}^{19)}$; the adenine base is not parallel to the uracil base intramolecularly, and the dihedral angle is $81.7(3)^{\circ}$. However, an intermolecular stacking interaction is observed between these bases. The adenine and uracil bases translated by a diad screw axis form a parallel alignment, as shown in Fig. $5(\mathrm{C})$ : their dihedral angle is $5.7(3)^{\circ}$ and the average interplanar spacing is $3.40 \AA$. As is clear from this figure, this interaction is stabilized by a strong dipole-dipole coupling interaction, because the directions of the respective dipole moments of the uracil and adenine bases, which were calculated by the $\mathrm{CNDO} / 2$ method, are almost antiparallel to each other. Furthermore, electrostatic attractions play a role in the stabilization: $-0.283-0.368$ for $\mathrm{N}(1) \mathrm{A}-\mathrm{C}(4) \mathrm{U}, 0.214--0.352$ for $\mathrm{C}(2) \mathrm{A}-\mathrm{O}(4) \mathrm{U}, 0.269--0.236$ for $\mathrm{C}(6) \mathrm{A}-\mathrm{N}(3) \mathrm{U}$ and $-0.244-0.452$ for $\mathrm{N}(6) \mathrm{A}-\mathrm{C}(2) \mathrm{U}$ atomic pairs.

On the other hand, a weak intramolecular stacking interaction is observed between the uracil base and indole ring, as can be seen in Fig. 5(D). The dihedral angle between them is $28.6(3)^{\circ}$, and the average interplanar spacing is $3.95 \AA$. Since this spacing value is outside the 


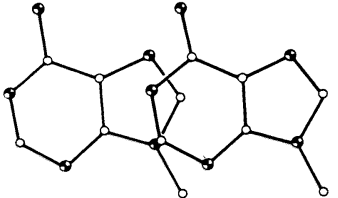

A

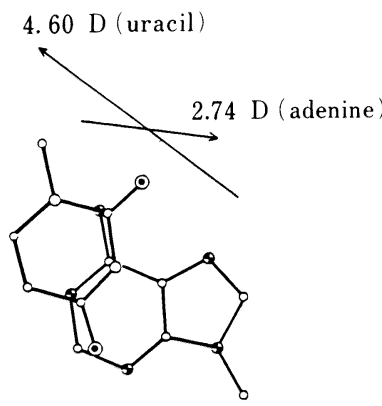

$\mathrm{C}$

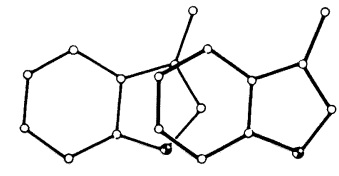

$\mathrm{B}$

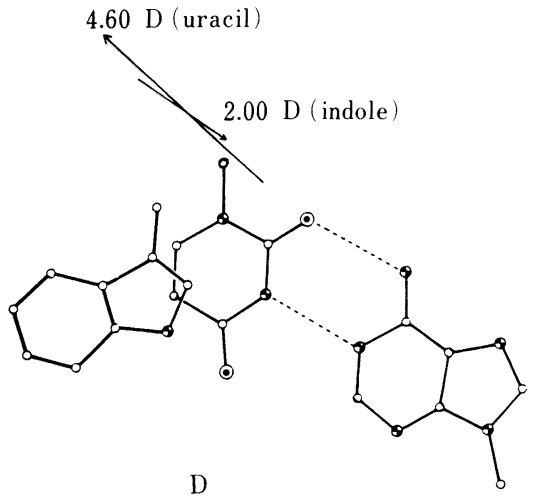

Fig. 5. Stacking Modes between Adenine-Adenine (A), Indole-Indole (B), Adenine-Uracil (C) and Uracil-Indole (D) Rings

Fig. 5(A) or (B) shows the projection perpendicular to the upper adenine or indole ring at $x, y, z$, and the lower adenine or indole ring is related by the symmetry operation of $x-1, y, z$, respectively. Fig. $5(\mathrm{C})$ shows the stacking mode of adenine (at $x, y, z)$ and uracil (at $2-x, y-0.5,2-z$ ) bases, viewed perpendicular to the adenine ring. Fig. 5(D) is a projection perpendicular to the central uracil base (at $x, y, z$ ), showing the stacking interaction with the indole ring (at $x, y, z)$ and the hydrogen bonding with the adenine base (at $1-x, y+0.5,2-z$ ). In Fig. 5(C) and (D), the vectors of permanent dipole moments are represented by arrows. The unit of dipole moment is debye (D).

normal van der Waals separation distance $(=3.4 \AA)$, the stacking interaction would be stabilized by the van der Waals contacts between the two aromatic rings. Figure 5(D) shows that the dipole-dipole interaction is important in determining the mutual orientation between the indole ring and the uracil base. A consideration of space-filling models suggest that the observed stacking mode is reasonable, without any steric hindrance, as an interaction mode of tryptophan residue with the uracil base of a single-stranded nucleic acid. Therefore this result means that the dipole-dipole interaction may be an important driving force in placing these two molecules close to each other.

Do the present X-ray results mean that tryptophan interacts with uracil base in preference to adenine base? Several solution studies ${ }^{20,21)}$ have suggested a preference for the adenine base, and a theoretical calculations ${ }^{5)}$ has also suggested the existence of indoleadenine stacking interaction. One reason why there is no interaction between the indole ring and the adenine base in this compound, may be that there is a difference between the connecting environments of the uracil and adenine bases to tryptophan, i.e., U-CO-NH-and $-\mathrm{CO}-\mathrm{NH}-\mathrm{A}$. Therefore further studies using various model compounds are necessary to examine the stacking mode between the indole ring and nucleic acid base at the atomic level.

\section{References and Notes}

1) This report is part XVIII of "Structural Studies of the Interaction between Indole Derivatives and Biologically Important Aromatic Compounds." Part XVII: T. Ishida, M. Itoh, M. Doi, and M. Inoue, Chem. Pharm. Bull., 
34, 4899 (1986).

2) B. Pullman and A. Pullman, Proc. Natl. Acad. Sci. U.S.A., 44, 1197 (1958).

3) For example, see C. Helene and J. C. Maurizot, CRC Crit. Rev. Biochem., 10, 213 (1981); C. Helene and G. Lancelot, Prog. Biophys. Mol. Biol., 39, 1 (1982).

4) T. Ishida, M. Shibata, K. Fujii, and M. Inoue, Biochemistry, 22, 3571 (1983).

5) T. Ishida, H. Usami, and M. Inoue, Chem. Pharm. Bull., 31, 4220 (1983).

6) T. Ishida, S. Ibe, and M. Inoue, J. Chem. Soc., Perkin Trans. 2, 1984, 297.

7) T. Ueda and J. J. Fox, J. Org. Chem., 29, 1762 (1964).

8) N. J. Leonard and R. F. Lambert, J. Org. Chem., 34, 3240 (1969).

9) P. Main, S. E. Hull, L. Lessinger, G. Germain, J. P. Declercq, and M. M. Woolfson, "A System of Computer Programs for the Automatic Solution of Crystal Structures from X-Ray Diffraction Data, MULTAN 78," University of York, England and Louvain, Belgium, 1978.

10) Tables of observed and calculated structure factors, anisotropic thermal parameters of nonhydrogen atoms, and atomic coordinates of hydrogen atoms are available from one of the authors (T.I.) on request.

11) T. Ashida (ed.), "The Universal Crystallographic Computing System-Osaka," Library of Programs, Computing Center, Osaka Univ., 1979.

12) D. T. Cromer and J. T. Waber, "International Tables for X-Ray Crystallography," Vol. 4, ed. by J. A. Ibers and W. C. Hamilton, Kynoch Press, Birmingham, 1974, p. 71.

13) D. Voet and A. Rich, Prog. Nucleic Acid. Res. Mol. Biol., 10, 183 (1970).

14) T. Ishida, Ph. D. Thesis, Osaka University, 1979, p. 125.

15) E. Benedetti, "Peptides, Proceedings of the 5th American Peptide Symposium," ed. by M. Goodman and J. Meienhofer, J. Wiley and Sons, New York, 1977, p. 257.

16) O. Bakke and A. Mostad, Acta Chem. Scand., B34, 559 (1980).

17) S. S. Tavale, T. D. Sakore, and H. M. Sobell, J. Mol. Biol., 43, 375 (1969).

18) J. A. Pople and G. A. Segal, J. Chem. Phys., 44, 3289 (1966).

19) N. C. Seeman, J. M. Rosenberg, F. L. Suddath, J. J. P. Kim, and A. Rich, J. Mol. Biol., 104, 109 (1976).

20) F. Morita, Biochim. Biophys. Acta, 343, 674 (1974).

21) K. G. Wagner and R. Lawaczeck, J. Mag. Res., 8, 164 (1972). 